of about 100 compared with the decay process of the rare isotope ${ }_{19} K^{40}$, and the disagreement would be still more emphasised. We know further that an isotope ${ }_{19} \mathrm{~K}^{42}$ cannot be responsible for the natural $\beta$-activity of potassium, for this isotope was produced artificially by Hevesy 4 and its half-period found to be about 16 hours. Therefore one comes to the conclusion that another relatively rare isotope of potassium exists, which is the source of the $\beta$-particles. This can only be ${ }_{19} \mathrm{~K}^{43}$, which is transformed by the decay process to the well-known isotope ${ }_{20} \mathrm{Ca}^{43}$. This seems to be the only assumption not in conflict with all the available experimental evidence.

K. Sirte.

Department of Physies,

German University,

Prague.

July 18 .

${ }^{1}$ G. Gamow, Nature, 133, 869; 1934. G. Beck and K. Sitte, NATURE, 133, 722; 1934.

Roy, Soc., A, 145, 638. 1935

hys., 91, $361 ; 1934$. K. Sitte, ibid., in print.

4. Hevesy, NATURE, 135, $96 ; 1935$.

\section{Spectroscopy of an Enzyme Reaction}

IF a suitable amount of monoethyl-hydrogen peroxide is added to an active preparation of liver catalase at $p H$, the enzyme spectrum disappears instantly, and two new absorption bands in the green range of the spectrum appear. In the course of a few minutes, at room temperature, the new absorption bands decrease in intensity, and simultaneously the original enzyme spectrum reappears. When the change is complete, the cycle may be repeated by the addition of fresh substrate. The time required for the reaction cycle at $4^{\circ}$ is four times that required at $24^{\circ}$. The reappearance of the enzyme spectrum is accompanied by the disappearance of the titratable peroxide oxygen from the system. No gas is evolved in this reaction. Acetaldehyde and ethyl alcohol are among the most probable reaction products.

The spectrum of the intermediate compound is to be attributed to a combination of the enzyme with the substrate. The alternative explanation that one is dealing here with the reduced (ferrous-) form of catalase ean be discarded because neither molecular oxygen nor ferricyanide reoxidise it to the original ferric form, and because it does not combine with carbon monoxide.

Enzyme spectrum (after K. Zeile') :

$$
\text { I. } 650 \ldots \underbrace{646-620}_{629} \cdots \underbrace{510-490}_{500} \text { m } \cdots \text {; II. } \underbrace{550-530}_{540} \cdots 520 \ldots
$$

Spectrum of the enzyme-substrate compound:

$$
\text { I. } \underbrace{576-564}_{570} \text {; II. } \underbrace{540-529}_{5345} \mathrm{~m} \mu \text {. }
$$

$5 \times 10^{5}$ molecules of the substituted peroxide per enzyme molecule are required to complete the transformation into the intermediate compound. It is interesting to note that according to J. B. S. Haldane's calculations', based on Zeile's and Euler's data, $1.9 \times 10^{5}$ molecules of hydrogen peroxide are destroyed by one molecule of liver catalase per second under optimal conditions. This coincidence is still more striking since the enzyme is at least 5,000 times more active towards hydrogen peroxide than .towards ethyl-hydrogen peroxide.
With hydrogen peroxide as a substrate, no apparent change of the enzyme spectrum is observed. The inference is, that since the rate of formation and the stoichiometry of the two enzyme-substrate complexes seem to be similar, the different catalytic efficiency towards the two substrates is due to the different velocity constants of the breakdown of the intermediate compounds.

A non-enzymatic model of the present observation is found in the reaction of methæmoglobin with hydrogen peroxide (Kobert ${ }^{3}$, Haurowitz ${ }^{4}$ ) and with monoethyl-hydrogen peroxide. Here the intermediate compounds are similar to that observed above, so far as the absorption spectrum is concerned. But one molecule of methæmoglobin will only break up $3 \cdot 6 \times 10^{-2}$ molecules of hydrogen peroxide per second (cf. K. G. Stern ${ }^{5}$ ), and an excess of only 20 molecules of hydrogen peroxide (Haurowitz, l.c.) and of 8 molecules of monoethyl-hydrogen peroxide will suffice to transform completely the spectrum of this catalyst into that of the intermediate compounds.

Courtauld Institute of Biochemistry, KurT G. Stern.

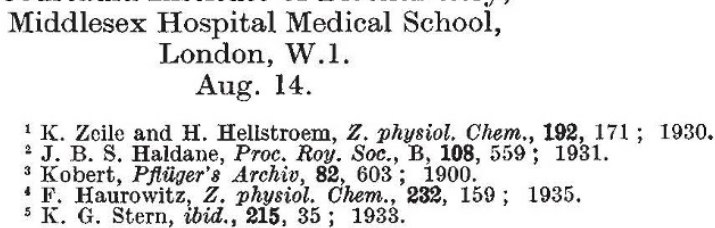

Pleochroism of Crystals of Rare-Earth Salts as Evidence of the Non-Rotation of Certain Electronic Orbits

So long ago as $1888, \mathrm{H}$. Becquere ${ }^{1}$ recorded that the absorption spectrum afforded by erystals of 'didymium' salts was different for different vibration planes of the incident light. Later, M. H. Dufet ${ }^{2}$ made a detailed study of the crystallographic and optical properties of neodymium, praseodymium and samarium sulphates, in the course of which he confirmed and extended Becquerel's observations by recording the wave-lengths of bands in the absorption spectra of these salts for light vibrating parallel to the acute bisectrix, obtuse bisectrix and optic binormal respectively. He found that the intensity of individual absorption bands was often, though not invariably, different for the three principal vibration planes, even to the extent of their being absent from one of them.

In the course of a study of the relationship between the optical properties and chemical constitution of certain absorbing crystals by means of a grating microspectrograph used in conjunction with a polarising microscope ${ }^{3}$, I have had occasion to examine the absorption spectra for various vibration directions in crystals of neodymium, praseodymium and samarium sulphates, and in ceric ammonium sulphate. The method adopted was to project a conoscopic ('directions') image of the crystal on the slit of the microspectrograph by means of a Bertrand lens, using a polariser but no analyser, and ensuring that the slit and the vibration plane of the polariser were parallel to a principal vibration plane of the crystal. An oil-immersion objective of $N \cdot A .=1 \cdot 30$, in conjunction with an oil-immersion condenser, was used.

In this way it was possible to obtain absorption spectra which showed the effect of varying the ray 